\title{
Morphologie des rapports sociaux des migrant(e)s
}

Un ménage roumain à Genzano (province de Rome)

Ionela Vlase

\section{(2) OpenEdition}

Journals

Édition électronique

URL : https://journals.openedition.org/remi/3761

DOI : 10.4000/remi.3761

ISSN : $1777-5418$

Éditeur

Université de Poitiers

\section{Édition imprimée}

Date de publication : 30 juin 2007

Pagination : 163-179

ISBN : 978-2-911627-44-6

ISSN : 0765-0752

Référence électronique

Ionela Vlase, " Morphologie des rapports sociaux des migrant(e)s », Revue européenne des migrations internationales [En ligne], vol. $23-\mathrm{n}^{\circ} 1$ | 2007, mis en ligne le 30 juin 2010, consulté le 16 avril 2022.

URL : http://journals.openedition.org/remi/3761 ; DOI : https://doi.org/10.4000/remi.3761

Ce document a été généré automatiquement le 16 avril 2022.

(c) Université de Poitiers 


\title{
Morphologie des rapports sociaux des migrant(e)s
}

\author{
Un ménage roumain à Genzano (province de Rome)
}

\author{
Ionela Vlase
}

1 Depuis l'effondrement du communisme en 1989 des formes de mobilité inconnues jusque là se sont développées en Roumanie. Malgré son caractère récent l'émigration roumaine est déjà diversifiée dans ses formes, ses objectifs, ses pays de destination. Elle a déjà attiré l'attention des différent(e)s chercheurs et chercheuses en sciences sociales qu'il s'agisse de la migration pendulaire des paysans roumains de l'Oaş en France (Diminescu, 1999), de la circulation migratoire comme stratégie de vie des paysans du Sud de la Roumanie (Sandu, 2000), de la migration hautement qualifiée des Roumain(e)s à Toronto (Nedelcu, 2005), de la migration de retour réalisée ou projetée par les migrant(e)s originaire de Focşani ${ }^{1}$ (Cingolani, Piperno, 2005), plus récemment de la migration des enfants ${ }^{2}$.

2 Le but du présent article n'est pas de traiter de l'ampleur et des différentes formes de mobilité développées par les migrant(e)s roumain(e)s pendant ces quinze dernières années, car d'autres auteurs s'y sont déjà arrêtés (Sandu, 2002 ; Diminescu, Ohlinger, Rey, 2003). Toutefois, il est certainement utile de rappeler le contexte migratoire particulier dans lequel cette étude s'inscrit, à savoir la migration roumaine en Italie, en tant que "champ migratoire ${ }^{3}$ " spécifique qui se distingue des autres champs migratoires - «français », « espagnol », « israélien », etc. (Diminescu, Ohlinger, Rey, 2003). Nous avons choisi de mettre en évidence des nouvelles formes de sociabilité développées dans un contexte migratoire spécifique, à savoir celui de la migration non qualifiée et souvent illégale qui transgresse les directions des mouvements classiques ${ }^{4}$. Cette recherche $\mathrm{e}^{5}$ a pour objectif d'analyser les logiques socio-économiques des différents types de ménages migrants roumains à Rome, ainsi que les rapports de genre et de génération à l'intérieur de ces formes hybrides de cohabitation. Le "ménage " - tel que nous le définissons plus loin - constitue une unité d'analyse située à l'intersection de problématiques comme la sociabilité et les réseaux migratoires, l'économie domestique et les rapports de genre. Ces ménages hybrides des migrant(e)s 
roumain(e)s à Rome sont à la fois, générés par et générateurs de réseaux migratoires. Connaissant l'importance des réseaux migratoires ${ }^{6}$ dans l'association des migrants roumains sous un même toit (Weber, 2004), et la structure par genre de ces réseaux migrants roumains (Potot, 2005), nous montrerons comment les ménages migrants sont structurés selon le genre et la génération et quels types de rapports s'établissent entre les membres de ces ménages. Il s'agira d'abord de rendre compte du contexte migratoire qui influence leur formation, puis de proposer une typologie des ménages migrants roumains selon l'étape historique de la migration. La dernière partie de ce texte sera consacrée à l'analyse détaillée d'un type de ménage migrant roumain à Rome afin de mettre en évidence les différents rapports sociaux entre les migrant(e)s qui en font partie.

\section{La migration roumaine en Italie}

\section{Le contexte à l'origine de l'émigration roumaine post-communiste}

3 L'immigration roumaine en Italie est surtout représentée par des originaires de Moldavie (ancienne province historique située au Sud-est de la Roumanie), en particulier des districts de Vrancea, Galat,i, Neamt, et Bacu. Selon le recensement communautaire sur la migration ${ }^{7}$ c'est l'Italie qui a attiré la grande majorité des émigrants : 78,6\% de ceux du district de Vrancea-milieu rural, $71,3 \%$ de ceux du district de Galat,i. La Turquie et la Grèce apparaissent pour ces districts comme des destinations secondaires, attirant 2 à $4 \%$ des originaires de ces districts (Sandu, 2002, selon les données du recensement communautaire 2001). La Moldavie, région la plus pauvre $d u$ pays se caractérise à l'époque post-communiste par une forte désindustrialisation des villes; des restructurations des entreprises peu productives et mal gérées ont laissé sans travail une grande partie des actifs, surtout parmi les hommes. Seule l'industrie textile se maintient dans la région. Très féminisée, elle est aussi très modestement rémunérée. Faute de moyens financiers pour survivre dans les villes, les gens sont retournés dans leurs villages d'origine, pratiquant une agriculture de subsistance et complétant leurs ressources par de courts séjours de travail (un à trois mois) dans les pays frontaliers comme la Turquie ou la Hongrie. Cette mobilité pendulaire, entre 1990 et 1993, a été qualifiée de "migration de tâtonnement" (Diminescu, Ohlinger, Rey, 2003). Elle sert d'apprentissage et de stratégie d'accumulation d'un capital financier indispensable pour ensuite franchir les frontières des pays européens plus éloignés. Diverses raisons expliquent leur préférence pour les pays du bassin méditerranéen et plus particulièrement pour l'Italie. On notera d'abord la facilité d'entrer, de séjourner et de trouver du travail illégalement dans ces pays (Bonifazi, 2000). De nombreuses lois de régularisation promulguées par le gouvernement italien ${ }^{8}$ durant les deux dernières décennies, ont bénéficié aux Roumains, dont la loi Bossi-Fini de 2002. De 95834 en 2002, les Roumain(e)s en possession d'un permis de séjour sont passé(e)s à 239426 en 2003 (Ricci, 2006).

De plus l'Italie pratique une politique de quotas annuels de nouveaux migrants ${ }^{9}$, qui visibilise les besoins en main-d'œuvre et facilite une insertion économique rapide, même en l'absence de papiers (Ambrosini, 1999). La forte demande de personnes travaillant dans le domaine de l'assistance aux personnes âgées (badanti) (Bimbi, 1993; Bettio et al, 2006) ou dans le secteur domestique (collaboratrice familiare) est un facteur 
d'attraction important pour l'immigration féminine. Les Roumaines y trouvent aussi une opportunité d'avoir un revenu et une possibilité d'apprendre la langue dans un cadre informel, souvent caractérisé à la fois par l'exploitation et la protection (Andall, 2003; Ambrosini, 1999). Par rapport à d'autres immigrées, qui ont beaucoup de difficulté à sortir du secteur domestique (Andall, 2003), les femmes roumaines trouvent relativement vite une alternative à l'occupation domestique, s'employant par la suite dans la restauration ou le commerce ou dans le secteur de la santé.

5 L'attraction de l'Italie est renforcée par l'image qu'ont les migrant(e)s roumain(e)s d'origine rurale de l'Italie, pays latin à la même aire culturelle et religieuse que la Roumanie. Leur faible niveau de capital culturel et surtout leur méconnaissance des langues étrangères les amènent à valoriser cette situation.

Il est intéressant de souligner que les femmes de la région moldave migrent proportionnellement plus souvent que celles des autres régions roumaines. Dans certains villages elles sont plus nombreuses que les hommes à partir.

"Je me demandais pourquoi les femmes migrent de plus en plus en Italie..."

"Alors, cela se passe comme ça parce que maintenant, ou disons que depuis une année et demie, on trouve plus de places de travail pour les femmes. Cela veut dire que maintenant, lorsqu'on part d'ici, de chez nous, on sait ce qui nous attend. Par exemple, ma petite sceur qui était déjà en Italie, c'est elle qui m'a prêté l'argent pour partir. Je dois lui rendre 1600 \$ car maintenant on peut aller et rentrer avec 200 euros, mais moi je suis partie avant le $1^{\text {er }}$ janvier 2002. [...] J'ai changé de places de travail ${ }^{10}$.» (Ana, 40 ans, originaire du village Vulturu, interviewée en décembre 2002 à Vulturu)

7 La ségrégation de genre sur le marché du travail en Italie a été montrée pour les migrant(e)s en général (Anthias et Lazaridis, 2000 ; Andall, 2003, Caritas, 2005) et les Roumain(e)s en particulier (Sandu, 2002 ; Vlase, 2004a). Les hommes travaillent dans la construction $^{11}$, soit de manière autonome soit dépendant d'un employeur italien, alors que les femmes travaillent dans le secteur domestique, les soins et le commerce. Dans tous les cas, les réseaux sociaux des migrant(e)s, fondés sur des liens de parenté directe et indirecte, d'amitiés et de voisinage constituent le principal moyen pour trouver une place dans ces secteurs caractérisés par l'informalité. Mais les réseaux sociaux s'avèrent également importants dans la formation des ménages migrants, compte tenu du déficit de logements dans les grandes villes italiennes et la difficulté des immigrés à accéder à un logement, surtout lorsqu'ils sont en situation irrégulière (Dell'Olio, 2004).

\section{La découverte d'un terrain : Genzano (province de Rome)}

8 La population roumaine migrante en Italie est concentrée surtout au centre du pays (plus de $40 \%$ ) suivi des régions du Nord-ouest et Nord-est (respectivement 31,4 \% et $21,4 \%$ ). Ainsi, les Roumain(e)s représentent $22 \%$ de l'ensemble des immigrés dans le Piémont et $21 \%$ (soit 70000 personnes) dans le Latium (Ricci, 2006). Une recherche menée par Cingolani e Piperno sur les réseaux migratoires transnationaux roumains met en lumière dans cette région la forte concentration des Roumains au Sud et au Sudest de la province de Rome, dans les quartiers de la capitale ainsi que dans les communes qui lui sont proches. La présence roumaine est remarquable aussi dans communes comme Genzano, Frascati, Aprilia, Pomezia, Nettuno situées entre 30 et $70 \mathrm{~km}$. de la capitale. La concentration de communautés migrantes dans des communes de faible taille n'est pas forcement le résultat de l'attractivité du marché du travail local. C'est vraisemblablement le fonctionnement des réseaux migratoires qui ont 
facilité l'installation et la recherche de travail des nouveaux arrivés, les liens sociaux qui se sont créés qui ont contribué à de telles concentrations dans des communes dans lesquelles des migrants d'autres nationalités sont peu représentés.

9 Afin d'illustrer quelques types de rapports sociaux entre les migrant(e)s roumain(e)s je m'appuie sur une étude de cas $^{12}$, celui d'un ménage de migrant(e)s roumain(e)s vivant à Genzano. Pourquoi ai-je choisi une telle unité d'analyse? D'abord parce que les migrants roumains n'ont pas encore eu le temps de fonder des associations facilement identifiables et observables. En effet la migration roumaine en Italie n'a commencé qu'au milieu des années 1990 ; de plus peu de migrants ont eu accès au permis de séjour avant les régularisations (sanatoria) de 1998 et de 2002. Leur statut irrégulier n'a pas facilité leur visibilité et la formation d'associations.

Dans les statistiques de l'INSAT, qui comptabilisent les immigrants réguliers, les Roumains ne figuraient pas en 1992 parmi les 12 premières communautés immigrées du pays. Flux migratoires et régularisations expliquent qu'en 2004 ils constituent première communauté immigrée du pays, avec 244377 personnes possédant un permis de séjour, soit $11 \%$ de l'ensemble des immigrés réguliers [ISTAT (Istituto nazionale di statistica), 2005].

11 Le choix du ménage comme unité d'analyse est motivé aussi par la densité d'échanges sociaux entre les immigrant(e)s dans ce cadre : la plupart des relations sociales entre les migrants se tissent autour du lieu de travail, du logement qu'ils se partagent et des règles qui gèrent ce partage. Ce choix s'avère intéressant puisqu'il permet de comprendre les logiques intimes selon lesquelles les migrant(e)s roumain(e)s se rassemblent pour faire face à un contexte nouveau, difficile du point de vue psychologique et socio-économique. C'est après le recueil des données que je me suis rendue compte de l'importance d'utiliser le "ménage migrant ${ }^{13}$ comme une unité d'analyse des rapports sociaux, alors que l'enquête de départ n'avait pas été formulée en ce sens. Le projet initial était d'expliquer les différents parcours migratoires des femmes et des hommes du village de Vulturu à la province de Rome, les stratégies et les moyens mobilisés par ces acteurs migrants, l'accès inégal des femmes et des hommes aux ressources leur permettant de migrer, etc. L'expérience de plusieurs semaines passées dans un ménage migrant roumain, ainsi que des observations spontanées dans d'autres ménages de la province et les données recueillies lors d'entretiens sur les formes de cohabitation m'ont permis de reconstruire le puzzle des rapports sociaux des migrant(e)s. Ce type de recherche a peu de précédents, exceptée la recherche de Serge Weber $^{14}$ (2004) qui porte sur la recomposition des maisonnées villageoises roumaines, en illustrant également l'association, sous le même toit, des différents noyaux de familles se connaissant depuis le village d'origine. L'auteur note: «nous observons des formes intéressantes de cohabitation. Il est rare qu'un logement soit occupé par une seule famille. Dans la commune d'Alatri, tous les logements que nous avons visités sont partagés entre plusieurs cellules de parenté accueillant entre trois et sept personnes. Les cellules associées sont presque familiales, mais rarement conformes au modèle de la famille restreinte. » (Weber, $2004: 40)$

12 Notre étude tente d'approfondir cette question, afin de déterminer les différents types de ménages et les facteurs du contexte migratoire qui influence la composition et la durabilité de ces associations d'immigrant(e)s roumain(e)s sous le même toit, au-delà de leur référentiel qui est la $"$ maisnie $»^{15}$, à savoir le groupe domestique traditionnel roumain comprenant deux à trois générations : les parents, les enfants célibataires et le 
dernier fils avec sa famille (l'épouse et les enfants) qui vivent sous le même toit et se partagent les produits de la terre et du bétail (Stahl, 2000).

Avant de présenter notre étude de cas, il faut décrire notre terrain: Genzano. Cette petite ville d'environ 30000 habitants, située à $40 \mathrm{~km}$ au Sud-est de Rome, héberge quelques centaines de Roumain(e)s, dont une bonne partie provient du village de Vulturu. Ce village, appartenant au district de Vrancea, compte actuellement quelques 4000 habitants, presque 2000 de moins que lors du recensement de 1992. Grâce aux réseaux migratoires construits sur les liens de parenté et de voisinage, une grande partie des villageois s'est concentrée à Genzano.

S'il est bien connu que la migration roumaine vers l'Italie commence vers 1994, on sait moins que la structure par genre des flux migratoires roumains, du moins ceux qui partaient du village de Vulturu, a enregistré des changements. Nous pouvons identifier trois étapes :

- De 1994 à 1998 la migration entre Vulturu et la province de Rome est quasi-exclusivement masculine. Le prix du visa touristique s'élève à environ 1000 \$ somme que seuls les hommes peuvent se procurer par des emprunts, par des ventes de biens ou, pour les jeunes mariés, par la dot en argent reçue à l'occasion de leur mariage, (pratique fréquente avant la suppression des visas pour les Roumains). Les femmes, elles, ne disposent d'aucun support financier pour migrer (Vlase, 2004a, 2004b). Elles ne peuvent pas non plus rejoindre leurs maris ou leurs pères au titre du regroupement familial à cause de la situation irrégulière de ceux-ci. Durant cette période deux opérations de régularisation des sans-papiers ont eu lieu en Italie : en 1995 et en 1998. La première venait trop tôt pour que les Roumain(e)s puissent en profiter et la deuxième n'a donné l'occasion qu'à quelques personnes de Vulturu de faire la demande de permis de séjours.

- De 1999 à 2002 la migration des femmes augmente par le biais du regroupement ou en utilisant un visa touristique. La féminisation de la migration roumaine est importante dans tout le pays, et en particulier à Vulturu.

- Depuis 2002 il est devenu courant que les femmes migrent en Italie indépendamment de leurs maris ou de leurs pères. Dans la région roumaine moldave en général et dans le village de Vulturu en particulier, les femmes qui migrent vers la province de Rome sont plus nombreuses que les hommes.

À partir de nos données de terrain, nous pouvons constater qu'à ces différentes étapes de la migration rurale roumaine en Italie correspondent, sur le plan social, des modèles de cohabitation des migrant(e)s. Ils sont influencés, d'une part, par les contraintes socio-économiques et politiques qui agissent sur les statuts des migrant(e)s et, d'autre part, par la référence au groupe domestique roumain traditionnel. Ces facteurs sont donc déterminants des nouvelles formes de sociabilité migratoire que nous avons étudiées à travers la cohabitation des immigrant(e)s dans la province de Rome.

\section{Les ménages des migrant(e)s roumain(e)s en Italie}

La population étudiée a connu et connaît encore des formes de cohabitation très diverses. Le ménage roumain rural traditionnel qui était encore assez courant dans le village de Vulturu est de plus en plus remis en question avec la migration féminine ${ }^{16}$ (Vlase, 2004a). Ainsi, en Italie, les migrant(e)s roumain(e)s ont dû réinventer de nouveaux types de ménages qui devaient répondre à de nouvelles contraintes socioéconomiques spécifiques au contexte migratoire. En même temps, les relations sociales 
- qu'elles soient d'amitié, de voisinage, de parenté ou simplement de connaissances qui existaient avant l'arrivée en Italie, connaissent des transformations ${ }^{17}$. Certaines peuvent se renforcer, tandis que d'autres peuvent aussi se défaire sous l'influence de nouvelles contraintes dans un contexte migratoire fragile. Si les premiers migrants roumains arrivés à Rome dormaient dans la gare de Termini ou chez leurs employeurs, petit à petit ils ont trouvé des maisons ou des appartements qu'ils habitaient à plusieurs afin de partager les frais. Leur manière de se mettre ensemble suivait une certaine logique que nous essayons de mettre en évidence à travers l'analyse d'un ménage.

\section{Les étapes de l'organisation des ménages}

17 De nos observations sur le terrain ainsi que des données recueillies par des entretiens, on peut dégager trois types de "ménages ", qui se succèdent pour chacun dans l'histoire migratoire.

18 Un type de ménage rudimentaire et marqué par une forte instabilité correspond à celui composé généralement par des hommes habitant une maison abandonnée sans aucun confort (sans électricité, eau courante, souvent sans fenêtres et portes). Le nombre des migrants qui cohabitent est très élevé - une dizaine, voire bien plus. S'il est difficile d'appeler cette forme de cohabitation « ménage », quelques règles de conduite générale s'imposent cependant :

... Je suis allé chez mon témoin ${ }^{18}$. Il habitait dans une maison abandonnée; il y avait une trentaine de gens là. La plupart de ces maisons sont en périphérie de la ville, mais il y en a aussi plus près du centre. Je ne sais pas ce qui s'est passé... peut-être quelqu'un avait-il juste un enfant et la maison était trop grande... peut-être d'autres n'avaient-ils plus l'argent pour les entretenir, ces maisons... mais il y a beaucoup de maisons abandonnées là-bas qui sont maintenant à des Roumains... ou à des immigrants (il rit)... là où j'ai habité il y avait un propriétaire. Cet homme, avant que les Roumains n' y arrivent, élevait du bétail dans la maison. Il a eu deux Roumains qui s'occupaient de ses animaux pour pouvoir y habiter sans payer, après quoi, quand les Roumains sont venus nombreux là, il a enlevé ses animaux et nous a "loué» la maison. On y était 30-40 personnes dont les trois quarts de Vulturu. Il y avait 4-5 pièces et on logeait 6 à 8 personnes dans une pièce. (Gigi, 33 ans, parti à Rome en 1994, interviewé à Vulturu le 17.12.2000)

19 Il est rare de trouver aujourd'hui une telle forme de cohabitation qui correspond à la première étape migratoire décrite plus haut.

Un autre type de ménage est constitué par les membres d'une famille élargie qui ne seraient pas forcément regroupés de la même façon dans leur village d'origine. Ce type de ménage résulte des regroupements familiaux que certains migrants ont réalisés une fois les papiers obtenus auxquels se rajoutent ensuite d'autres parents (beaux-frères, tantes, cousins, etc.). Ce type de ménage est beaucoup plus stable et, du point de vue du statut juridique, "semi régularisé " - les personnes qui n'ont pas bénéficié du regroupement familial, même si elles sont membres de la famille (élargie) sont souvent des migrant(e)s sans papiers. On a là un type de ménage plus complexe du point de vue relationnel, car les femmes et les enfants en font déjà partie.

21 Le troisième type, sur lequel porte notre étude de cas, présente un mélange des deux types précédents. Il n'est ni étendu comme dans le premier cas, où des dizaines de personnes cohabitent, ni limité aux membres de la famille élargie. Son principal intérêt est sa tendance à ressembler à une famille. 


\section{Faire semblant d'être une « famille » : l'étude d'un ménage à Genzano}

L'étude de ce ménage qui a traversé d'importants changements, met en évidence certains rôles et certains critères d'association des migrant(e)s dans une situation fragile, vulnérable et dont ils essaient de se sortir au mieux. Mon entrée dans ce ménage a été facilitée par la connaissance de celui qui en est «leader». Si dans le ménage traditionnel roumain le chef du ménage est l'homme le plus âgé du groupe domestique, dans le ménage issu de l'immigration, ce rôle est difficile à attribuer et à faire reconnaître par les autres membres du ménage, car il ne repose pas sur une définition exacte des statuts et des tâches à remplir, il est plutôt en permanente négociation. Toutefois, quelques critères conduisent à se doter d'un «leader ». Il s'agit de la personne qui assume le logement, qui signe le bail, qui s'engage à régler les loyers, la distribution des pièces et la répartition des tâches pour chaque personne. Outre ces responsabilités, le « leader » du ménage doit avoir aussi une certaine autorité reconnue par les autres membres du ménage. Cette autorité peut reposer sur une expérience réussie de la migration, sur une bonne connaissance des opportunités et des risques en tant que migrant irrégulier, voire sur une formation professionnelle plus élevée que celle des autres membres du ménage, ou tout autre aspect qui pourrait être considéré comme un atout dans le contexte migratoire. Parfois il y a des négociations, des disputes et même des ruptures lorsque ce rôle n'est pas reconnu par tous. L'importance du leader est primordiale dans le fonctionnement et la durée d'un ménage, car sans une autorité reconnue, ses membres ont tendance à se quereller rapidement. Compte tenu de la difficulté de trouver un logement en tant que migrant, et surtout en tant que migrant irrégulier, il est bien entendu que les migrant(e)s roumain(e)s ont intérêt à vivre le plus longtemps possible sous un même toit et à faire en sorte que le ménage ne se délite pas.

Pour définir le ménage, on retiendra trois éléments principaux : un espace commun à habiter, un ensemble de personnes avec une origine commune (le même district, voire le même village), et la gestion en commun des tâches ménagères et des frais du loyer, $\mathrm{y}$ compris des charges.

\section{Construction du ménage}

Quand j'ai rencontré ce ménage en arrivant à Genzano il regroupait quatre personnes, trois hommes et une femme, qui partageaient un appartement spacieux de trois pièces. L'histoire du ménage m'a été racontée par Ricardo ${ }^{19}$. En arrivant à Rome, il a été accueilli par ses cousins germains, deux frères qui partageaient un studio avec d'autres migrants roumains.

J'ai essayé à droite et à gauche, j'ai demandé de l'aide aux collègues, aux connaissances, aux Italiens. Mon cousin, le cadet, qui travaillait dans une boulangerie, a trouvé une place pour moi seulement le 2 janvier. Donc j'ai attendu 2 mois sans rien faire... tu comprends que si on ne gagne rien et qu'on ne contribue au bien-être de la maison, du ménage, déjà les choses prennent d'autres tournures, les discussions interviennent... mais ensuite on s'est bien remis sur pieds. (Ricardo, 34 ans, arrivé le 15 octobre 1997, originaire de Vulturu, interviewée à Genzano le 1er mars 2003) 
Il raconte en même temps que ce logement a offert un abri à beaucoup de gens qui sont passés par là, dont certains pour des périodes courtes ou simplement en transit : avait une petite mansarde à l'étage, où on pouvait aussi dormir. C'est là que j'ai habité moi, ... ou plutôt moi aussi, pendant les deux premières années. Plein de monde était passé par là, différentes personnes sont venues, à un moment donné on était huit dans cette pièce. En général il y avait des personnes avec différents degrés de parenté ou amis, même des connaissances.

Après avoir habité deux ans avec ses cousins, Ricardo a déménagé chez un ancien ami d'enfance qui avait aussi habité un temps dans la maison des cousins de Ricardo.

Je te le dis exactement à partir du moment où je suis arrivé, avant je ne sais pas ce que c'était; je suis arrivé trois ou quatre mois après que Carlo ait pris cette maison. À ce moment-là, il y avait une famille qui habitait cette chambre-là, il y avait Marina avec encore une personne dans l'autre pièce, et dans ma pièce il y avait Carlo et encore un garçon qui travaillait de nuit. Donc on était sept personnes dans cet appartement.

Ce ménage à sept n'a pas duré et au bout de quelques mois, il s'est entièrement transformé : quelques personnes ont quitté la maison, d'autres ont créé des conflits, puis des problèmes de paiement du loyer et de l'électricité, ont participé à une détérioration de l'habitat. De plus, l'absence d'un «chef de ménage » reconnu ou la revendication de cette position par plusieurs personnes a été la cause de la transformation du ménage. Ricardo nous dit que même les relations d'amitié en ont souffert :

Je suis retourné chez mes cousins, où j'avais habité la première fois, dans le même studio, et cela puisque l'amitié de trente ans (envers Carlo) s'est avérée être nulle. Je regrette, mais les gens (les Roumains) changent ici, lorsqu'ils arrivent en Italie.

Le nouveau contexte n'est pas sans incidence sur les rapports sociaux des migrants. Certains liens peuvent être renforcés tandis que d'autres peuvent s'affaiblir. La migration est un test pour le contenu et la force de ses relations entre les migrants. Dans le cas où la composition d'un ménage se maintient plus d'une année en l'absence même d'un degré de parenté entre les membres, de nouveaux rapports sociaux se créent selon des règles bien fixées dans le cadre du ménage. Dans ce cas-là, le rôle du «leader» du ménage est important pour sa stabilité. Dans notre étude de cas par exemple, il n'y a pas de relations de parenté (à part les deux frères qui habitent ensemble la plus petite pièce de l'appartement). Tout est négocié et créé sur place et c'est ce processus qui revêt des significations pour notre analyse. Le portrait des personnes contribue à éclairer leur position dans la structure des rôles dans le cadre du ménage.

29 Ricardo, âgé de 34 ans a une formation universitaire d'ingénieur qu'il a achevée en Roumanie en 1995. Il est arrivé à Rome en 1997.

«La première chose que j'ai faite: ... j'ai dormi, ensuite j'ai essayé de m'habituer à la vie d'ici, de chercher un travail ; j'ai travaillé ici et là... les premières expériences au travail ont été : cueillir les olives et les kiwis... c'est ce qu'on trouve le plus souvent ici, dans cette région, car, comme je suis arrivé le 15 octobre, c'était leur période de récolte. Donc, j’ai travaillé environ une semaine... ou plus précisément cinq jours à la récolte des olives et ensuite une semaine à la récolte des kiwis et après rien d'autre. J'ai réalisé alors que ce n'est pas ça la façon de vivre en Italie, que cela ne peut pas t'assurer un niveau de vie décent. " (Ricardo)

Ricardo est le second enfant d'une famille relativement aisée de Vulturu. Sa sœur aînée est mariée et vit avec la famille de son mari (selon la coutume). Ses parents, anciens instituteurs actuellement à la retraite, sont restés au village. Après les quatre 
premières années de séjour en Italie sans retourner au village, il a fait trois courts séjours chez ses parents. Il n'est pas marié et ses parents ont hâte de le voir fonder une famille. Il est passionné par la musique et essaie de se faire remarquer dans ce domaine. Suite à la recomposition du ménage décrite plus haut, il a contacté le propriétaire de l'appartement et l'a convaincu qu'il pourrait mieux le gérer. Il occupe la plus grande pièce, environ $25 \mathrm{~m} 2$ de l'appartement de Genzano. Il possède des instruments de musique (guitare, orgue) et un ordinateur. Il travaille dans une boulangerie, aux côtés d'une dizaine de migrants roumains, pour la plupart originaires de Vulturu.

Marina est originaire de la ville de Focani. Âgée de 39 ans, elle est arrivée à Genzano en 1999 chez ses frère et belle-sœur qui habitaient déjà cette même maison. Elle est mariée et son mari et sa fille de 18 ans, qui termine ses études secondaires, sont restés au pays. Faute de papiers, elle n'est pas retournée dans sa famille pendant quatre ans. En Roumanie, elle avait travaillé comme gestionnaire d'une boutique de journaux. À Rome, elle avait trouvé, après six mois d'attente, une place comme domestique logée, qu'elle a quitté pour un emploi dans la restauration grâce à l'aide de sa belle-sœur. Après des tensions avec celle-ci, son frère et sa femme ont quitté la maison. Elle occupe une pièce de $20 \mathrm{~m} 2$.

Le loyer est de $650 €$ et on partage à quatre, c'est-à-dire le nombre de personnes qui habitent ici. Cela ne veut pas dire que les personnes qui habitent dans les autres pièces ne peuvent pas entrer dans ma chambre. Elles peuvent entrer, cela ne me dérange pas pour autant qu'elles ne touchent pas à mes affaires [...]. Ensuite, chacun nettoie sa chambre, évidemment... Comme on a deux salles de bains, l'une est utilisée par Marina, car c'est normal, elle est la seule femme, et l'autre par nous, les hommes. Et pour le reste on nettoie chacun tour à tour. (Ricardo)

Bien qu'ils ne se soient pas rencontrés avant cette cohabitation, Marina et Ricardo semblent s'entendre très bien sur l'organisation de la maison. Elle nettoie souvent les espaces communs ainsi que de la chambre de Ricardo. De même qu'elle cuisine pour lui et parfois pour les deux frères :

C'est normal que je lui serve le repas. Moi,j'ai un frère et j'ai toujours fait comme ça pour lui. Mais comme il a quitté la maison avec sa femme, il me manque maintenant. Je fais pareil pour Ricardo car je trouve qu'il est comme un frère pour moi. Souvent on m'a demandé comment je peux habiter avec tous ces hommes, mais je réponds toujours qu'il est plus facile que d'habiter avec des femmes... (Marina)

Les deux frères, Mario, le cadet, âgé de 34 ans et Silvio, l'aîné, âgé de 39 ans, habitent la troisième pièce de l'appartement, la plus petite $(15 \mathrm{~m} 2)$. Ils viennent de Vulturu et avaient fréquenté la même école primaire. Mario a suivi l'école d'apprenti soudeur et travaille en Italie dans la même boulangerie que Ricardo. Il est marié et son épouse et leurs deux filles vivent avec ses parents (la cadette n'était pas née au moment où Mario est parti en Italie). Depuis son départ en 1999, il n'a pas pu rentrer au village, car il est encore sans papiers bien qu'en attente de les obtenir. Il n'était au village ni pour la naissance de sa cadette ni pour la mort de son père.

J'ai beaucoup pleuré et la seule chose qui m'a consolé est le fait que je pouvais leurs envoyer de l'argent. Je ne suis pas venu pour rester toute ma vie parmi les Italiens. Je suis venu pour gagner leur argent et puis rentrer chez moi.

Silvio est arrivé en Italie en 1998 et a été accueilli par ses neveux originaires du même village. Il a fait les mêmes études que son frère, et a travaillé à Bucarest. Sa femme et sa fille y habitent toujours. Au moment où je suis arrivée à Genzano, il était rentré pour quelques semaines dans sa famille. Lors de mon deuxième séjour en septembre 2003, j'ai eu l'occasion de le connaître. Plus tard il a fait venir sa famille en Italie et l'a 
temporairement installée dans cette même maison, le temps de chercher un nouvel appartement.

Aujourd'hui le ménage migrant qui fait l'objet de cette étude est composé de Ricardo, Marina et sa fille qui l'a rejointe après la fin de ses études, et de deux autres personnes (père et fille) qui ont repris la chambre des frères. Silvio et Mario ont donc quitté l'appartement, le premier lorsqu'il a fait venir sa famille en Italie, et a cherché un nouveau logement, le deuxième lorsqu'il est parti dans le Nord de l'Italie pour travailler. Cependant, la composition du ménage de quatre personnes, dont nous avons parlé plus haut, a été relativement stable, ne connaissant pas de changements pendant environ trois ans. Quelles sont les raisons d'une telle stabilité, alors que les ménages roumains migrants à Rome changent de composition au moins une fois par an?

\section{La recherche du consensus à l'intérieur du ménage}

Comme nous l'avons précisé chacune des personnes qui composaient le ménage était introduite dans la maison par quelqu'un qui y résidait déjà. Ricardo, devenu responsable pour le propriétaire de la maison, décidait de l'entrée de nouveaux membres. On rappellera que Ricardo ${ }^{20}$ est le plus instruit et qu'il est le premier à avoir été régularisé par son employeur italien. La composition du ménage a changé au cours de son histoire, comme nous l'avons vu et toutes les séparations ou regroupements que le ménage a connu reposaient sur le type de rapports sociaux qui existaient entre les migrant(e)s et sur la manière dont les rôles étaient répartis entre les membres. Les relations déjà existantes au village peuvent se perpétuer ou se détériorer en migration, alors que les migrants disposent de faibles ressources, que des migrants sans travail doivent se faire entretenir par les autres, qu'il est difficile de partager entre plusieurs personnes et familles une seule maison, plus ou moins bien équipée. Ils doivent gérer ces ressources de la façon la plus efficace pour pouvoir s'en sortir, ce qui demande au quotidien de la coopération et de l'entraide, mais ce qui permet aussi de minimiser les coûts de la vie (loyer, nourriture) et de recevoir un soutien moral (le besoin de parler, d'écouter, d'être conseillé etc.).

Cette analyse pourrait laisser croire que le fonctionnement d'un tel ménage est très proche de ce que représente une «famille » (famille nucléaire ou famille étendue). Mais les ménages de migrants roumains à Rome ne sont pas exactement des familles. Ils sont plutôt des unités beaucoup moins durables, qui peuvent se défaire et se refaire sur une période parfois assez courte. En outre, les liens entre les membres qui composent un ménage à un moment donné sont divers: de parenté, de voisinage, d'amitié ou de connaissances (des personnes qui se connaissent à peine ou qui font connaissance grâce au ménage improvisé). Ce qui est intéressant à remarquer à propos du cas que nous avons présenté, c'est que les conflits interviennent entre parents, alors que les liens peuvent être jugés comme forts. Dans la situation décrite c'est Marina qui va essayer de rompre avec ses proches (son frère et sa belle-sœur) pour renforcer les liens avec Ricardo.

Le choix de Marina est un choix rationnel. Si l'on tient compte de sa situation irrégulière à Rome, elle souhaite au moment où elle fera la demande d'un permis de séjour, disposer d'un hébergement sûr (condition importante pour faire un dossier). Comme Ricardo a déjà des papiers et une grande maison, il peut la déclarer comme colocataire. C'est ce qu'il fera aussi pour les deux frères Silvio et Mario. Ce cas illustre le rôle des liens faibles entre les migrant(e)s (Granovetter, 1983). En se repliant 
exclusivement sur les liens de parenté, ils risquent de ne pas avoir accès à un système élargi d'informations et d'entraide. L'arrivée à Rome suppose de leur part un investissement personnel afin de mieux connaître, apprivoiser ou maîtriser ce nouveau lieu. Les relations et les échanges avec les autres Roumain(e)s à Rome constituent un moyen essentiel pour y arriver. Ceux et celles qui n'ont pas de telles relations ont intérêt à les créer, ceux et celles qui en ont quelques-unes ont intérêt à les garder, les renforcer et les multiplier. De tels liens peuvent se créer en cohabitant dans une maison.

\section{Les convergences dans les divergences des formes de ménage}

Le type de ménage décrit est un ménage mixte d'adultes à d'âge actif (moins de 50 ans, en général). La présence des enfants et des retraités est rare, voire inexistante car cela entraîne des frais supplémentaires sans contrepartie. Comme Ricardo nous le fait comprendre: "Nous partageons les frais au nombre de personnes adultes qui habitent la maison. Si quelqu'un amène son enfant, on ne peut pas lui demander de payer». Silvio a dû chercher un autre logement lorsque sa femme et sa fille sont venues en Italie. Ce type de ménage ne ressemble pas au modèle du ménage traditionnel qui est généralement organisé sur trois générations. En revanche, la présence des femmes dans un ménage est souhaitée car elles assurent souvent la propreté de l'habitat et dans certains cas la cuisine. Le contenu et le nombre des tâches varient d'un ménage à l'autre selon la position de la femme dans celui-ci (Pessar, 1995). Parfois, si son mari fait aussi partie du ménage, ces tâches se limitent au bien-être du couple. Si le mari n'est pas présent, comme dans le cas de notre exemple, la femme cuisine et nettoie pour tout le ménage. Mais la présence de plus d'une femme dans un tel ménage peut être aussi une source de conflits. Comme on l'a vu, les disputes entre les deux belles-sœurs vont conduire à la séparation: le couple marié va quitter l'appartement, tandis que Marina restera avec les autres. Cette remarque sur l'effet du genre dans le fonctionnement d'un ménage est importante car c'est un élément qui permet également de comprendre les nouveaux rapports sociaux en migration. La présence du mari dans le ménage migrant exerce une influence sur le pouvoir de la femme de négocier son indépendance par rapport aux autres membres du ménage. Certaines fois, les femmes peuvent ainsi accomplir plus de tâches (faire le ménage et le repas pour tous les autres membres du ménage surtout si ces derniers sont des parents ou amis du mari) et d'autres fois, en s'appuyant sur l'autorité de leur mari dans le ménage, mieux négocier leur indépendance.

Si au niveau des dépenses ménagères les choses paraissent bien claires, il n'en est pas de même en ce qui concerne la distribution des tâches (faire les repas, nettoyer les espaces communs). La réponse de Ricardo à ma question portant sur la distribution des tâches commence par mettre en évidence le fait que Marina dispose d'une salle de bain pour elle seule, tandis que les trois hommes utilisent tous la seconde salle de bain. Il dit aussi qu'il y a une distribution égalitaire des tâches, mais les observations faites au cours des semaines passées dans ce ménage ont montré le contraire. Marina assume une très grande partie des tâches ménagères tout en rendant plus de services à Ricardo qu'aux frères qui habitent dans la maison. En même temps, elle justifie son attitude envers ses co-locataires par les relations différentes avec chacun. Elle considère Ricardo comme beaucoup plus proche d'elle. 
41 La plupart des liens sociaux entre les Roumain(e)s de la province de Rome se construisent à partir des relations déjà existantes avant la migration, ainsi la plupart des personnes à Genzano viennent du même village ou des localités situées à proximité. Ces relations vont petit à petit changer avec les nouvelles contraintes et les ressources limitées auxquelles les migrants, pour la plupart illégaux, sont confrontés. Notre recherche a montré que des gens qui se connaissent depuis l'enfance ou qui se connaissent moins, voire pas du tout, arrivent ensemble dans la même maison et doivent faire fonctionner un ménage à plusieurs. C'est une situation qui n'aurait jamais été envisageable dans leur lieu d'origine. Une telle cohabitation est d'autant plus difficile quand elle met ensemble des gens très différents, des femmes et des hommes, des jeunes et des personnes âgées, des personnes avec des niveaux différents de formation, dont le seul but commun est la recherche d'un travail. Ce mélange très hétérogène rend la cohabitation parmi les plus intéressantes du point de vue des relations qui s'y tissent. L'espace d'une maison devient le terrain d'échanges, de conflits ou d'enjeux qui contribuent tous à la re-définition des liens sociaux entre les migrant(e)s.

Malgré les nombreuses différences entre les ménages migrants roumains à Rome et le modèle traditionnel d'organisation du groupe domestique au pays, nous pouvons constater aussi quelques similitudes. D'ailleurs ce sont ces convergences qui assurent la longévité des ménages migrants, par la solidarité mutuelle qui s'installe entre ses membres. Tout en gardant son rôle et les devoirs envers sa famille d'origine, chacun d'entre eux essaie d'assumer, dans le cadre du ménage migrant un rôle qui peut correspondre à une position différente de celle du groupe domestique d'origine. Un migrant peut ainsi continuer à être père de famille pour ses enfants restés au village et assumer un rôle de frère dans le nouveau ménage. Il y a évidemment des situations où un mari ayant laissé sa femme et ses enfants au village joue le rôle du mari dans le nouveau ménage migrant. Les conflits des rôles dans une telle situation conduisent tôt ou tard à des séparations. À la différence des études sur les ménages migrants roumains à Rome (Weber, 2004) qui montrent que ces ménages durent plus longtemps lorsqu'il y a des rapports de parenté entre les membres du ménage (qu'ils précèdent la migration ou se créent après la mise en ménage), nous constatons que c'est plutôt la manière de distribuer les tâches et les rôles à l'intérieur du ménage qui jouent, faisant ainsi qu'il ressemble au groupe domestique d'origine.

\section{BIBLIOGRAPHIE}

AMBROSINI Maurizio (1997) Alla scoperta della diversità. Un panorama dell'immigrazione in Italia, in Ambrosini Maurizio, Meri Salati éds., Il valore della differenza. Tendenze, problemi, interventi sull'immigrazione straniera, Milano, Torino : Paoline, pp. 12-66.

AMBROSINI Maurizio (1999) Travailler dans l'ombre. Les immigrés dans l'économie informelle, in Revue Européenne des Migrations Internationales, (15) 2, pp. 95-121. 
ANDALL Jacqueline (2003) Hierarchy and interdependence: the emergence of a service cast in Europe in Andall Jacqueline éd., Gender and Ethnicity in contemporary Europe, Oxford, New York: Berg, pp. 39-60.

ANTHIAS Floya, LAZARIDIS Gabriela (eds) (2000) Gender and migration in Southern Europe, Oxford, New York: Berg, 263 p.

BETTIO Francesca, SIMONAZZI Annamaria, VILLA Paola (2006) Change in care regimes and female migration "care drain" in the Mediterranean, Journal of European social policy, vol. 16 (3), pp. 271-285.

BIMBI Franca (1993) Gender "gift relationship" and welfare state cultures in Italy, in Jane Lewis (éd.) Women and social policies in Europe: work, family and the state, UK, USA: Edward Elgar, pp. 138-169.

BONIFAZI Corrado (2000) European migration policy: questions from Italy, in Russel King, Gabriela Lazaridis and Charalambos Tsardanidis eds., Eldorado or fortress? Migration in Southern Europe, pp. 235-252.

CAMPANI Giovanna (1999) La politique migratoire italienne : contrôle des frontières, régularisation et intégration, Cahiers de l'URMIS : Les politiques de l'immigration (Unité de Recherche Migrations et Société), n 5, pp. 47- 58.

CARITAS (2005) Immigrazione. Dossier Statistico 2005, Roma: Nuova Antarem.

CODINI Ennio (2003) Regulatory aspects, in The eighth Italian report on migrations 2002, Milan: ISMU, pp. 35-51.

CINGOLANI Pietro, PIPERNO Flavia (2005) «Il prossino anno a casa ». Radicamento, rientro e percorsi translocali: il caso delle reti migratorie Marginea-Torino e Focşani-Roma, Programma Migraction 2004-2005, Occasional paper, CeSPI, Fieri.

Dell'OLIO Fiorella (2004) Immigration and immigrant policy in Italy and the UK: is housing policy a barrier to a common approach towards immigration in the EU?, Journal of ethnic and migration studies, vol. 30 (1), pp. 107-128.

DIMINESCU Dana (2003) Visibles mais peu nombreux. Les circulations migratoires roumaines, Paris, MSH, 409 p.

DIMINESCU Dana, OHLINGER Rainer, REY Violette (2003) Les circulations migratoires roumaines : une intégration européenne par le bas ? Cahiers de recherche de la MIRE, $\mathrm{n}^{\circ}$ 15, pp. 61-69.

DIMINESCU Dana (2004) La mobilité des jeunes roumains à l'heure de l'intégration européenne, Hommes et migrations : « Enfants sans frontières », septembre-octobre 2004, n 1251, pp. 42-50.

ESPINOSA Kristin and MASSEY Douglas (1999) Undocumented migration and the quality and quantity of social capital in Ludger Pries ed., Migration and transnational social spaces, pp 106-137.

GLUCKMAN Max (1961) Ethnographic data in British social anthropology, Sociological Review 9 , pp. 5-17.

GRANOVETTER Mark (1983) The strength of weak ties: A network theory revisited in Sociological theory, vol. 1, pp. 201-233.

ISTAT (2005) Gli stranieri in Italia. Gli effetti dell'ultima regolarizzazione. Stima al 1genaio 2005.

Statistiques en bref, www.istat.it/salastampa/comunicati/non calendario/20051215 00/testointegrale.pdf 
KLEINMAN Sherryl, COPP Martha (1993), Emotions and fieldwork, Newbury Park, London, New Delhi: Sage publications, $69 \mathrm{p}$.

MARCUS George (1995) Ethnography in/of the world system: the emergence of multi-sided ethnography, Annual Review of Anthropology, vol. 24, pp. 95-117.

MIHAILESCU Vintil, NICOLAU Viorica (1995) Du village à la ville et retour. La maisnie mixte diffuse en Roumanie, Bulletin of the Ethnographical Institute SASA, vol. XLIV Beograd, pp. 77-84.

Ministère de la Solidarité Sociale (2006) Ulteriori ingressi di lavoratori stagionali extracomunitari per l'anno 2006, circulaire 24/2006, tiré le 21/06/2006 du lien

http://www.welfare.gov.it/Sociale/immigrazione+ed+integrazione/norme/circolari/ 20060810Circolaren.24del2006.htm

MITCHELL J. Clyde (2002 [1983]) Case and situation analysis, in Case study method, Roger Gomm et al. eds., Thousand Oaks, London, New Delhi: Sage Publications, pp. 165-186.

NEDELCU Mihaela (2005) Stratégies de migration et d'accès au marché de travail des professionnelles roumaines à Toronto : rapports de genre et nouvelles dynamiques migratoires, Revue Européenne des Migrations Internationales, (21) 1, pp. 77-106.

PESSAR Patricia (1995) On the homefront and in the workplace: integrating immigrant women into feminist discourse, Anthropological quarterly, vol. 68, $\mathrm{n}^{\circ} 1$, pp. 37-47.

POTOT Swanie (2005) La place des femmes dans les réseaux migrants roumains, Revue Européenne des Migrations Internationales, (21) 1, pp. 243-257.

RICCI Antonio (2006) I Romeni in Italia : immigrazione, radicamento e ritorno, in Tra Romania e Italia, Societatea Real, n 4, Bucarest: Paideia, pp. 25-53.

SANDU Dumitru (2000) Migrat,ia circulatorie ca strategie de viat,, Sociologie româneasc, $\mathrm{n}^{\circ} 2$, Bucarest, pp. 5-29.

SANDU Dumitru (2002) Migrat,ia transnat,ional a românilor din perspectiva unui recensmânt comunitar, Sociologie româneasc, $n^{\circ} 3-4$, pp. 5-52.

SANDU Dumitru (2005) Emerging transnational migration from Romanian villages, Current Sociology, vol. 53 (4), Sage publications, pp. 555-582.

STAHL, Paul H. (2000) Triburi si sate din sud-estul Europei, Bucuresti: Paideia.

STAKE, Robert E. (1995), The art of case study research, Thousand Oaks, London, New Delhi: Sage publications, $175 \mathrm{p}$.

VLASE Ionela (2004a) L'insertion des femmes roumaines sur le marché du travail à Rome : un moyen de développement personnel et collectif, in Reysoo F. et Verschuur C. (coord.), Femmes en mouvement. Genre, migrations et nouvelle division du travail, IUED Genève, pp. 115-126.

VLASE Ionela (2004b) Hommes et femmes en migration : d'un village roumain à Rome Migration/ Société, Paris-CIEMI, n 93-94, pp. 47-60.

WEBER Serge (2004) De la chaîne migratoire à la migration individuelle des Roumains à Rome, Hommes \& Migrations, $\mathrm{n}^{\circ}$ 1250, pp. 38-48.

ZEHRAOUI Ahsène (1994), L'immigration de l'homme seul à la famille, Paris : L'Harmattan, 180 p. 


\section{NOTES}

1. Ville roumaine située au Sud est du pays reconnue par son grand nombre de migrant(e)s séjournant et travaillant en Italie.

2. Différentes études sur la migration (de travail) des mineurs roumains en France ou en Italie ont été menée les deux dernières années afin de sensibiliser les institutions étatiques et les familles aux dangers qui guettent cette catégorie de migrant(e)s.

3. Ce concept a été utilisé pour dénommer un espace de circulation migratoire ayant des limites plutôt diffuses mais marqué par le choix d'une destination préférée par les migrant(e)s originaire de cet espace, alors que les régions migratoires sont des espaces mieux définis qui se structurent par la préférence des migrant(e)s pour plusieurs pays de destination (Sandu, 2002).

4. En rapport aux les anciens mouvements migratoires Sud-Nord, Est-Ouest, on constate aujourd'hui, surtout au niveau européen, des trajectoires plus complexes. Dans le cas présent, il s'agit d'un mouvement de l'Est au Sud de l'Europe.

5. L'étude est fondée sur une recherche de terrain inscrite dans un travail de thèse de doctorat et qui porte sur l'analyse de la migration de travail d'une population rurale roumaine en Italie. Dans ce but, trente entretiens semi-directifs (menés en roumain mais traduit en français par la suite) avec des femmes et des hommes migrant(e)s originaires du village de Vulturu (Sud-Est de la Roumanie) ont été menées, ainsi que des observations directes et des études de cas ont été réalisées lors des différents séjours de recherche dans le village et dans la province de Rome (la région d'accueil) entre 2000 et 2006.

6. L'importance des réseaux sociaux a été souvent mise en évidence à travers l'accès au marché du travail (Granovetter, 1983) ou dans la prédiction du comportement migratoire des femmes et des hommes (Espinosa et Massey, 1999), mais rarement les chercheurs se sont interrogés sur le lien entre les réseaux migratoires et l'accès au marché immobilier, ou la façon dont les migrant(e)s cohabitent un appartement.

7. Ce recensement a été réalisé par OIM Bucarest en collaboration avec le Ministère de l'Intérieur et le Ministère des Informations Publiques entre novembre et décembre 2001 et a porté sur presque tous les villages roumains (12 300 sur 12700 villages) ainsi que sur 151 petites villes (de moins de 20000 personnes). Les données concernant le nombre des personnes parties à l'étranger, les destinations principales de ces migrant(e)s, le nombre des retours, les occupations des migrant(e)s dans les pays de destination, l'utilisation de l'argent gagné à l'étranger, ont été recueillies sur la base d'un questionnaire distribué aux mairies des villages et rempli par les différents notables en tant qu'experts locaux.

8. Pour une analyse détaillée voir Campani (1999) et Codini (2003).

9. Le tout dernier en la matière est la circulaire 24/2006 qui annonce un quota de 30000 travailleurs extracommunautaires repartis selon les besoins des différentes régions italiennes.

10. Durant sa première expérience de migration de dix mois à Rome, Ana occupe successivement trois emplois de domestique, puis trouve une place dans un restaurant, toujours de façon irrégulière. Elle espère le retrouver si elle retourne en Italie.

11. Pour les migrants roumains originaires du milieu rural cette occupation concerne environ $45 \%$ des hommes, alors que l'agriculture connaît une baisse de $20 \%$ à $13 \%$ entre les premières vagues de migrant(e)s roumain(e)s et les dernières (Sandu, 2002).

12. L'analyse de ce cas a été possible grâce à une connaissance approfondie de ce ménage à l'intérieur duquel j'ai passé plusieurs semaines lors des séjours de recherche dans le département de Rome.

13. Par ménage migrant nous entendons la cohabitation d'un groupe de migrant(e)s dans un espace clos (maison, appartement) et l'organisation en commun des différentes tâches 
domestiques, sans que les membres soient nécessairement liés par des rapports de parenté directe ou indirecte.

14. Je remercie cet auteur pour les échanges d'idées intéressantes que nous avons eu lors des rencontres sur ce terrain commun d'étude.

15. Ce type de groupe domestique a connu une transformation - «la maisnie mixte diffuse » (Mihailescu et Nicolau, 1995) - durant le communisme, lorsqu'une partie des membres des ménages a migré vers les villes industrielles pour travailler dans les usines. Malgré la séparation physique, les auteurs mentionnés montrent qu'il y a une unité fonctionnelle entretenue par les réseaux diffus et les échanges denses entre les deux parties des ménages situées l'une dans le village et l'autre dans la ville. Cette unité a permis le regroupement des ménages après l'effondrement du communisme, lorsque la partie située en ville, poussée par les difficultés économiques, a rejoint celle restée au village.

16. Il est possible que le contexte socio-économique actuel en Roumanie ait eu une influence importante sur le changement du modèle traditionnel du groupe domestique rural défini plus haut, mais la migration, surtout celle des femmes, a constitué un accélérateur important de ces changements, par la rencontre des modèles domestiques différents dans la société d'accueil. Ainsi, d'autres modèles familiaux (notamment la famille nucléaire) apparaissent comme une meilleure alternative surtout aux yeux des migrantes roumaines

17. À ce propos nous notons que la plupart des auteurs insistent sur le renforcement des liens en migration, alors que sur le terrain nous avons pu remarquer aussi des tensions, des conflits et la rupture de relations d'amitié et de parenté.

18. Les témoins de mariage ont un rôle important dans les villages roumains orthodoxes. Les jeunes mariés choisissent un couple ou une famille généralement plus aisée qu'eux, qui tout au long de leur vie, accompagne la jeune famille lors des évènements importants, leur apporte aide et soutien en cas de besoin, baptise un ou plusieurs de leurs enfants etc. En migration, les témoins de mariage sont souvent ceux qui soutiennent les projets migratoires des jeunes mariés. 19. Toutes les personnes du ménage vont recevoir des surnoms.

20. Sur l'échantillon des 30 personnes interviewées (hommes et femmes confondus), c'est le seul migrant ayant un titre universitaire.

\section{AUTEUR}

\section{IONELA VLASE}

Assistante d'enseignement, Université de Neuchâtel, Institut de Sociologie, Faubourg de l'Hôpital 27 CH-2000 Neuchâtel, Suisse ; ionela.vlase@unine.ch 